\title{
ON REPRESENTATIONS OF A GENERALIZED METHOD OF FIELD QUANTIZATION
}

\author{
R. KLEEMAN
}

\begin{abstract}
A new method of field quantization, in which the number operator has the form $a^{*} a$, is proposed. Representations of this method are considered, particularly in reference to what conditions unique-vacuum state representations are required to satisfy.
\end{abstract}

\section{Introduction}

Over the past few years many different methods of generalized field quantization have been considered. The most prominent of these have been the parafield theories of Green [5], who assumes the following algebraic relations for the creation and annihilation operators $a_{i}^{*}$ and $a_{i}$ :

$$
\text { and } \left.\begin{array}{ll} 
& {\left[a_{i}, \frac{1}{2}\left[a_{j}^{*}, a_{k}\right]_{ \pm}\right]_{-}=\delta_{i j} a_{k}} \\
& {\left[a_{i},\left[a_{j}, a_{k}\right]_{ \pm}\right]_{-}=0,}
\end{array}\right\}
$$

with the + referring to para-Bose theories and the - to para-Fermi theories. In addition, para-Fermi theories, which have finite dimensional irreducible representations, have the following condition added:

$$
\left(a_{i}\right)^{p+1}=0 \quad \text { and } \quad(a)^{l} \neq 0 \text { for } l<p,
$$

the corresponding theories being referred to as para-Fermi statistics of order $p$.

The number operators for the para-field theories are taken to be

$$
N_{t}=\frac{1}{2}\left[a_{i}^{*}, a_{i}\right]_{ \pm}+b_{0}
$$

with the constant $b_{0}$ being fixed by the particular representation under consideration (for para-Fermi theories by the order of the theory).

(C) Copyright Australian Mathematical Society 1981 
Equations (1.1) may be justified in a free field theory context in the following manner. The field operator $\phi(x)$ is assumed to be given by

$$
\phi(x)=V^{-1 / 2} \sum_{j}\left[a_{j} \exp \left(i\left(k_{j}^{\mu} x_{\mu}\right)\right) u_{j}+b_{j}^{*} \exp \left(-i\left(k_{j}^{\mu} x_{\mu}\right)\right) v_{j}\right]
$$

where we are dropping any vector or spinor indices from $\phi(x)$. The $a_{j}$ are the annihilation operators for particles and the $b_{j}^{*}$ are creation operators for antiparticles. The $u_{j}$ and $v_{j}$ are appropriate normalizing coefficients for the discrete Fourier transform (1.4).

Secondly, we assume that the momentum-energy operator of the field is given by

$$
P^{\mu}=\sum_{j} k_{j}^{\mu} N_{j}
$$

Finally, we assume that Heisenberg's principle, in the following form, holds:

$$
\left[P_{\mu}, \phi(x)\right]=-i \partial_{\mu} \phi(x) \text {. }
$$

A short calculation from (1.4) and (1.5) now shows that, for this condition to hold, it is sufficient that

$$
\left[a, N_{k}\right]_{-}=\delta_{j k} a_{k} \quad \text { and } \quad\left[b_{j}^{*}, N_{k}\right]_{-}=-\delta_{j k} b_{k}^{*} .
$$

The adjoint to the first of these equations has the same form as the second and so we will remove the algebraic distinction between particle and antiparticle and simply write

$$
\left[a, N_{k}\right]_{-}=\delta_{j k} a_{k} \text {. }
$$

This equation merely expresses the fact that $a_{j}$ is an annihilation operator for the $j$ eigenstate of the field. Assumption of the form (1.3) for the number operator then gives:

$$
\left[a_{i}, \frac{1}{2}\left[a_{j}^{*}, a_{j}\right]_{ \pm}\right]_{-}=\delta_{i j} a_{j}
$$

The first of (1.1) has been obtained from (1.9) by Bialynicki-Birula [1] by making the assumption that the form of (1.9) should not be affected if the expansion functions of (1.4) are subject to a unitary transformation, the new annihilation operators after this transformation being given by

$$
a_{i}^{\prime}=\sum_{j}\left(\delta_{i j}+\alpha_{i j}\right) a_{j}
$$

where an infinitesimal unitary transformation is being considered and so $\alpha_{i j}=$ $-\alpha_{f i}^{*}$ is an anti-Hermitian matrix.

The second equation of (1.1) can be derived from the first by algebraic means.

The above justification for (1.1) is quite general, and it is only when one identifies the number operators with the expressions in (1.3) that one obtains the 
para-field theories. This raises the interesting question of whether other expressions for the number operators are possible.

A natural way of considering this possibility is to examine the expressions for the number operators of the usual Fermi and Bose quantization schemes. In both these cases they are assumed to be given by (see [6])

$$
N_{i}=a_{i}^{*} a_{i}
$$

By use of the anti-commutation and commutation relations

$$
\left[a_{i}^{*}, a_{j}\right]_{ \pm}=\delta_{i j} 1
$$

assumed for the Fermi and Bose cases, respectively, (1.11) becomes

$$
N_{i}=\frac{1}{2}\left[a_{i}^{*}, a_{i}\right]_{ \pm}-\frac{1}{2},
$$

which is in the form of (1.3).

From a purely formal viewpoint then, it appears natural to assume that only (1.11) holds. Equation (1.8) then becomes

$$
\left[a_{i}, a_{j}^{*} a_{j}\right]_{-}=\delta_{i j} a_{j}
$$

Such schemes have been considered before, most notably by Roman and Aghassi [7]. However, these authors also assume that the following commutation relations hold, with $p$ having the same role as in (1.2):

$$
\text { and } \left.\begin{array}{rl}
{\left[a_{i}^{*}, a_{j}\right]_{-}} & =\delta_{i j}\left(1-\Omega_{j}\right), \quad\left[a_{i}, a_{j}\right]_{-}=0 \\
\Omega_{j} & =[(p+1) / p !]\left(a_{j}^{*}\right)^{p}\left(a_{j}\right)^{p},
\end{array}\right\}
$$

which, as has been pointed out by Govorkov [4], are not invariant with respect to the infinitesimal unitary transformations (1.10). The requirement that (1.13) be invariant with respect to these transformations leads to [3]

$$
\left[a_{i}, a_{j}^{*} a_{k}\right]=\delta_{i j} a_{k} .
$$

We shall take this equation to be fundamental. In addition, we shall concern ourselves with only finite dimensional representations and so we will assume that (1.2) holds. This equation may be interpreted as a generalized exclusion principle: only $p$ particles being allowed in one particular eigenstate of the field.

In the appendix it is shown that a certain "truncated" oscillator considered by Buchdahl [2] has the same irreducible representations as our scheme with $i=j=k$. In the next section we consider properties of representations of the more general scheme. 


\section{Representations}

As stated in the introduction we shall be concerned with representations of the following scheme

$$
\left.\begin{array}{c}
{\left[a_{i}, a_{j}^{*} a_{k}\right]=\delta_{i j} a_{k}, \quad\left(a_{i}\right)^{p+1}=0,} \\
\left(a_{i}\right)^{l} \neq 0 \quad \text { for } l<p \quad \text { and } \quad N_{1}=a_{i}^{*} a_{i} .
\end{array}\right\}
$$

We begin by making a few general observations. First, we have $\left[N_{i}, N_{j}\right]=0$, meaning we can choose a basis for representations in which all number operators are diagonal. We shall denote by $\left|i_{1}, i_{2}, \ldots\right\rangle$ normalized eigenvectors of $N_{1}, N_{2}, \ldots$ which are in such a basis. These will be referred to as number states.

Secondly, we observe that the eigenvalues of $N_{i}$ are non-negative integers and finally that $a_{j}\left|i_{1}, i_{2}, \ldots, i_{j}, \ldots\right\rangle$ is an eigenvector of $N_{1}, N_{2}, \ldots$ with eigenvalues $i_{1}, i_{2}, \ldots, i_{j}-1, \ldots$ unless $i_{j}=0$ in which case it is zero. The last two assertions will be proved in the appendix.

Our first result on representations of (2.1) concerns degeneracy of the vacuum, that is, the existence of more than one linearly independent $|0,0, \ldots\rangle$ state:

THEOREM 1. If any of the number states are degenerate then the vacuum is degenerate.

Proof. Let $\phi$ and $\psi$ be two linearly independent $\left|i_{1}, i_{2}, \ldots, i_{l}, \ldots\right\rangle$ states; then, since they are normalized, $|(\phi, \psi)|^{1 / 2} \neq 1$. If $\omega$ is any state denote by $[\omega]$ a normalized state. Now

$$
\left\|a_{l} \phi\right\|=\left|\left(a_{l} \phi, a_{l} \phi\right)\right|^{1 / 2}=\left|\left(\phi, a_{l}^{*} a_{l} \phi\right)\right|^{1 / 2}=\left(i_{l}\right)^{1 / 2} .
$$

Hence

$$
\begin{aligned}
\left|\left(\left[a_{l} \phi\right],\left[a_{l} \psi\right]\right)\right|^{1 / 2} & =\left(i_{l}\right)^{-1 / 2}\left|\left(a_{l} \phi, a_{l} \psi\right)\right|^{1 / 2} \\
& =\left(i_{l}\right)^{-1 / 2}\left|\left(\phi, a_{l}^{*} a_{l} \psi\right)\right|^{1 / 2}=|(\phi, \psi)|^{1 / 2} \neq 1 .
\end{aligned}
$$

Thus $\left[a_{l} \phi\right]$ and $[a, \psi]$ are linearly independent, showing that there must exist more than one linearly independent $\left|i_{1}, i_{2}, \ldots, i_{l}-1, \ldots\right\rangle$ state. By applying the appropriate sequence of annihilation operators and using the above argument repeatedly, we conclude that the vacuum must be degenerate.

This result contrasts with the situation in para-Fermi theory in which degnerate number states are possible without a degenerate vacuum. The degeneracy of the number states in this theory is usually taken to mean that non-identical particles are described. If we accept this interpretation here, we must conclude that a representation of (2.1) with a unique vacuum state describes identical particles. 
It is possible to use conditions on the eigenvalues of $N_{1}, N_{2}, \ldots$ to separate the degenerate vacuum representations from the unique vacuum representations. These conditions are as follows:

THEOREM 2. If there exists $a\left|i_{1}, i_{2}, \ldots\right\rangle$ in the representation with

$$
\sum_{j} i_{j}>p \quad \text { and } \quad i_{m}>1 \text { for some } m,
$$

then the representation has a degenerate vacuum.

Proof. Let $\psi$ be a $\left|i_{1}, i_{2}, \ldots\right\rangle$ state satisfying the conditions of (2.3). Now, apart from $i_{m}$, there must exist an $i_{l} \neq 0$ as, otherwise, we will have $i_{m}>p$ which means, by the observations made at the start of this section, that $\left(a_{m}\right)^{p+1} \psi \neq 0$, contradicting the second of (2.1). Now the first equation of (2.1) gives

$$
a_{m} a_{m}^{*} a_{l} \psi-a_{m}^{*} a_{l} a_{m} \psi=a_{l} \psi
$$

If we assume that the first term on the left vanishes then $\left(a_{m}^{*} a_{l} a_{m} \psi, a_{l} \psi\right)=$ $-(a, \psi, a, \psi)$ and so $\left(a_{l} a_{m} \psi, a_{m} a_{l} \psi\right)=-i_{l}$. Using (2.2), $\left|\left(\left[a_{l} a_{m} \psi\right],\left[a_{m} a_{l} \psi\right]\right)\right|$ $=i_{l} / i_{l} \cdot i_{m}=\left(i_{m}\right)^{-1}$, which means that $\left|\left(\left[a_{l} a_{m} \psi\right],\left[a_{m} a_{l} \psi\right]\right)\right|^{1 / 2}=\left(i_{m}\right)^{-1 / 2} \neq 1$ and, since $\left[a_{l} a_{m} \psi\right]$ and $\left[a_{m} a_{l} \psi\right]$ have the same set of eigenvalues, it follows that the representation containing $\psi$ has a degenerate number state and consequently a degenerate vacuum.

If the first term of (2.4) does not vanish, then we have $a_{m}^{*} a_{l} \psi \neq 0$ which means, using the fact that $a_{m}^{*}$ is a creation operator for the $m$ th eigenstate, that there exists a $\psi^{\prime}$ in the representation with the eigenvalue set $i_{1}, i_{2}, \ldots, i_{m}+$ $1, \ldots, i_{l}-1, \ldots$.

Applying the above argument to $\left[\psi^{\prime}\right]$ with $l$ changed so that $i_{l} \neq 0$, and iterating this argument in the obvious way, we conclude eventually that if there is a unique vacuum state then there must exist a state for which $i_{l}=0$ for all $l \neq m$ which, as we have seen above, is not possible.

Thus the possible number states in unique vacuum state representations are those satisfying

$$
\sum_{j} i_{j}<p
$$

together with Fermi-like states which have $i_{n}=0,1$ for all $n$. That all number states satisfying (2.5) are allowable in unique vacuum state representations can be seen by considering the following representation. We define the action of $a_{l}$ and $a_{l}^{*}$ as follows:

$$
\begin{gathered}
a_{l}\left|i_{1}, i_{2}, \ldots, i_{l}, \ldots\right\rangle=\left(i_{l}\right)^{1 / 2}\left|i_{1}, i_{2}, \ldots, i_{l}-1, \ldots\right\rangle \\
a_{l}^{*}\left|i_{1}, i_{2}, \ldots, i_{l}, \ldots\right\rangle=\Delta\left(\sum_{j} i_{j}\right)\left(i_{l}+1\right)^{1 / 2}\left|i_{1}, i_{2}, \ldots, i_{l}+1, \ldots\right\rangle
\end{gathered}
$$


with $\Delta(x)=1$ for $x<p$, and $\Delta(x)=0$ for $x>p$. We also assume that there is a unique $\left|i_{1}, i_{2}, \ldots\right\rangle$ for each different choice of $i_{1}, i_{2}, \ldots$ satisfying $\Sigma_{j} i_{j}<p$.

It is an easy matter to show that (2.6) is a representation of (2.1). The Fermi-like states are allowable by virtue of the fact that Fermi-quantization is included in (2.1).

We now prove a result which gives a clearer idea of what the unique vacuum state representations of (2.1) are.

THEOREM 3. For $p>1$ all states satisfying the condition (2.5) are in unique vacuum state representations of (2.1). Furthermore these are the only states in such representations.

Proof. We begin by proving the first part of the result. First, we note that we need prove only that all states satisfying the equality of (2.5) are included. This follows since all other states may be obtained by an appropriate sequence of annihilation operators. We now prove two lemmas.

LeMmA 1 . If the state $\phi=\left|b_{1}, b_{2}, \ldots, b_{i}, \ldots, b_{k-1}, 0, \ldots\right\rangle$ exists in a representation with $b_{\imath} \neq 0$, then the state $\phi^{\prime}=\left|b_{1}, b_{2}, \ldots, b_{i}-1, \ldots, b_{k-1}, 1, \ldots\right\rangle$ does also.

Proof. Apply $\left[a_{k}, a_{k}^{*} a_{i}\right]=a_{i}$ to $\phi$. Then $a_{k} a_{k}^{*} a_{i} \phi-a_{k}^{*} a_{i} a_{k} \phi=a_{i} \phi$, and so $a_{k} a_{k}^{*} a_{i} \phi=a_{i} \phi \neq 0$. Thus $a_{k}^{*} a_{i} \phi \neq 0$, meaning that $\phi^{\prime}$ exists.

Lemma 2. If the states $\phi^{1}=\left|b_{1}, b_{2}, \ldots, b_{i}+1, \ldots, b_{k}-1, \ldots\right\rangle$ and $\phi^{2}=$ $\left|b_{1}, b_{2}, \ldots, b_{i}, \ldots, b_{k}, \ldots\right\rangle$, with $b_{i}, b_{k} \neq 0$, are in the representation, then the state $\phi^{3}=\left|b_{1}, b_{2}, \ldots, b_{i}-1, \ldots, b_{k}+1, \ldots\right\rangle$ is also.

Proof. We introduce the following notation:

$$
\begin{aligned}
\phi^{4} & =\left|b_{1}, b_{2}, \ldots, b_{i}-1, \ldots, b_{k}, \ldots\right\rangle, \\
\phi^{5} & =\left|b_{1}, b_{2}, \ldots, b_{i}-1, \ldots, b_{k}-1, \ldots\right\rangle, \\
\phi^{6} & =\left|b_{1}, b_{2}, \ldots, b_{i}, \ldots, b_{k}-1, \ldots\right\rangle .
\end{aligned}
$$

We now note that if $a_{l} \phi^{k}=\mu \phi^{m}$ then $a_{l}^{*} \phi^{m}=\bar{\mu} \phi^{k}$. This follows since $\left\|a_{l} \phi^{k}\right\|=$ $q^{1 / 2}$, where $a_{l}^{*} a_{l} \phi^{k}=q \phi^{k}$, meaning that $a_{l} \phi^{k}=\tau q^{1 / 2} \phi^{m}$, with $|\tau|=1 . q \phi^{k}=$ $a_{l}^{*} a_{l} \phi^{k}=a_{l}^{*} \phi^{m} \tau q^{1 / 2}$, which means $a_{l}^{*} \phi^{m}=\bar{\tau} q^{1 / 2} \phi^{k}$, as required. Thus we can 
introduce the following relations:

$$
\begin{array}{rlrl}
a_{i} \phi^{2} & =\alpha \phi^{4} ; & a_{i}^{*} \phi^{4}=\bar{\alpha} \phi^{2} ; \\
a_{k} \phi^{2}=\beta \phi^{6} ; & a_{k}^{*} \phi^{6}=\bar{\beta} \phi^{2} ; \\
a_{i} \phi^{6}=\gamma \phi^{5} ; & a_{i}^{*} \phi^{5}=\bar{\gamma} \phi^{6} ; \\
a_{i}^{*} \phi^{6}=\delta \phi^{1} ; & a_{i} \phi^{1}=\bar{\delta} \phi^{6} ; \\
a_{k} \phi^{4}=\varepsilon \phi^{5} ; & a_{k}^{*} \phi^{5}=\bar{\varepsilon} \phi^{4} .
\end{array}
$$

In addition, we have $\alpha \bar{\alpha}=b_{i}, \beta \bar{\beta}=b_{k}, \gamma \bar{\gamma}=b_{i}, \delta \bar{\delta}=b_{i}+1$ and $\varepsilon \bar{\varepsilon}=b_{k}$. Because of these relations, it is evident that all $\phi^{k}$ exist except possibly for $\phi^{3}$. We assume that it does not and arrive at a contradiction. We apply $\left[a_{k}^{*} a_{i}, a_{i}^{*}\right]=$ $a_{k}^{*}$ to $\phi^{6}$, obtaining $\delta \bar{\delta} \bar{\beta}-\gamma \bar{\varepsilon} \bar{\alpha}=\bar{\beta}$, and so $\beta=\bar{\gamma} \varepsilon \alpha / b_{i}$. Secondly, we apply $\left[a_{k}, a_{k}^{*} a_{i}\right]=a_{i}$ to $\phi^{2}$, noting that $\phi^{3}$ is assumed not to exist. This gives $-\beta \gamma \bar{\varepsilon}=\alpha$. Upon substituting the result for $\beta$ above, we obtain $b_{k}=-1$, which is clearly not possible, and so the lemma is proved.

We now note that the third equation of (2.1) implies that the following state must be in a representation: $|0,0, \ldots, p, 0,0, \ldots\rangle$, with the $p$ occurring in an arbitrary position. Suppose now we have an arbitrary state $\left|c_{1}, c_{2}, \ldots, c_{i}, \ldots\right\rangle$ satisfying $\Sigma_{i} c_{i}=p$. Further suppose that $c_{i}$ is the first non-zero eigenvalue of the state and $c_{k}$ the second. Then, by virtue of Lemmas 1 and 2 , the following sequence of states must exist:

$$
\begin{gathered}
\left|c_{1}, c_{2}, \ldots, c_{i-1}, p, \ldots, c_{k-1}, 0, \ldots\right\rangle, \\
\left(c_{1}, c_{2}, \ldots, c_{i-1}=0 ; c_{i+1}, \ldots, c_{k-1}=0\right) \\
\left|c_{1}, c_{2}, \ldots, c_{i-1}, p-1, \ldots, c_{k-1}, 1,0, \ldots\right\rangle, \\
\left|c_{1}, c_{2}, \ldots, c_{i-1}, p-c_{k}, \ldots, c_{k-1}, c_{k}, 0,0, \ldots\right\rangle .
\end{gathered}
$$

Assume that $c_{l}$ is the next non-zero eigenvalue of the state; then the existence sequence continues as

$$
\left|c_{1}, c_{2}, \ldots, c_{1-1}, p-c_{k}, \ldots, c_{k-1}, c_{k}, c_{k+1}, \ldots, c_{l-1}, 1,0, \ldots\right\rangle
$$

with $c_{k+1}, \ldots, c_{l-1}=0$,

$$
\left|c_{1}, c_{2}, \ldots, c_{i-1}, p-c_{k}-c_{l}, \ldots, c_{k-1}, c_{k}, c_{k+1}, \ldots, c_{l-1}, c_{l}, 0, \ldots\right\rangle .
$$

By continuing this procedure it is easy to see that the arbitrary state belongs to the representation.

We now prove the second part of the result. From the discussion of allowable states given above it is evident that we need show only that a Fermi-like state not satisfying the condition (2.5) is not allowable in the representation. Suppose $\phi=\left|b_{1}, b_{2}, \ldots\right\rangle$ is such a state and it is contained in the representation. Then, by applying an appropriate sequence of annihilation operators, it follows that $\phi^{\prime}=\left|c_{1}, c_{2}, \ldots\right\rangle$ exists with $\Sigma_{i} c_{1}=p+1$. Now let $k \neq l \neq m$ be such that 
$c_{k}=c_{l}=c_{m}=1$, and apply $\left[a_{k}, a_{l}^{*} a_{m}\right]=0$ to $\phi^{\prime}$ to get $a_{k} a_{l}^{*} a_{m} \phi^{\prime}=a_{l}^{*} a_{m} a_{k} \phi^{\prime}$. The left hand side of this expression vanishes because of (2.5). The right hand side, however, cannot vanish since, if it did, then there would have to be two states with $c_{m}=c_{k}=0, c_{l}=1$ (with all other $c_{i}$ 's identical with those for $\phi^{\prime}$ ) one which $a_{l}^{*}$ sent to zero and the other which $a_{l}^{*}$ would send to a state with $c_{m}=c_{k}=0, c_{l}=2$ (again with the other $c_{i}$ 's identical with those of $\phi^{\prime}$ ) which must exist because of the first part of our result. This situation is not possible without a degenerate vacuum, and so we have a contradiction.

We now prove an interesting result concerning degenerate vacuum representations:

THEOREM 4. There exists a degenerate number state which contains three vectors not scalar multiples of each other. These vectors are obtainable by applying creation and annihilation operators to one eigenvector $\phi$ of the representation. Furthermore, the vectors obey a linear relation and so span a 2-dimensional space only.

Proof. In proving Theorem 2, we essentially proved that there must exist an eigenvector in the representation with $i_{t} \neq 0$ and $i_{m}>1$ such that the first term on the left hand side of (2.4) vanishes. Let this vector be $\phi$. Hence $\left[a_{l} a_{m} \phi\right]$ and $\left[a_{m} a_{l} \phi\right]$ are not scalar multiples of each other. (They are also non-zero by the final observation at the beginning of this section.) Now (2.5) implies that

$$
-a_{m}^{*} a_{l} a_{m} \phi=a_{l} \phi \text {. }
$$

Furthermore, (2.1) shows that $a_{l}\left(a_{m} \phi\right)=a_{m} a_{m}^{*} a_{l}\left(a_{m} \phi\right)-a_{m}^{*} a_{l} a_{m}\left(a_{m} \phi\right)$ and, using (2.7),

$$
a_{l}\left(a_{m} \phi\right)=-a_{m} a_{l} \phi-a_{m}^{*} a_{l} a_{m} a_{m} \phi .
$$

Now the second term on the right cannot vanish as, otherwise, $\left[a_{m} a_{l} \phi\right]$ and $\left[a_{l} a_{m} \phi\right]$ would be scalar multiples. Also it cannot be a scalar multiple of either of these vectors for the same reason. Finally, it belongs to the same number state as $\left[a_{l} a_{m} \phi\right]$ and $\left[a_{m} a_{l} \phi\right]$ by observation of its form.

By using the proof of Theorem 1, it is clear that the degenerate vacuum also has the property of Theorem 4.

\section{Existence of representations}

A question that needs to be asked is the following. Does the algebraic system (2.1) have any representation apart from the obvious Fermi representation of $p=1$ ? The answer to this question is clearly affirmative. For the case of the 
unique vacuum state situation, (2.6) implicitly defines such a representation and it is not hard to see how this definition could be made completely explicit. For the case of a degenerate vacuum we present the following example. As yet the author has not been able to obtain a simple ansatz for obtaining such representations explicitly. We present the representation diagramatically in Figure 1.

The dots in the diagram represent normalized state vectors, the arrowed lines show the action of $a_{j}^{*}, j=1,2$, on states at the base of the lines, while the unarrowed lines represent both the actions of $a_{j}^{*}$ and $a_{j}$. The factors beside the lines give the factor by which the resultant state is multiplied after the action of $a_{j}^{*}$ and $a_{j}$ on the initial state. That this diagram is indeed a representation of (2.1) can be verified quite simply, albeit tediously. It is perhaps worthwhile pointing out that Theorem 4 of Section 2 applies to the degenerate states of the diagram which are consequently all linearly dependent.

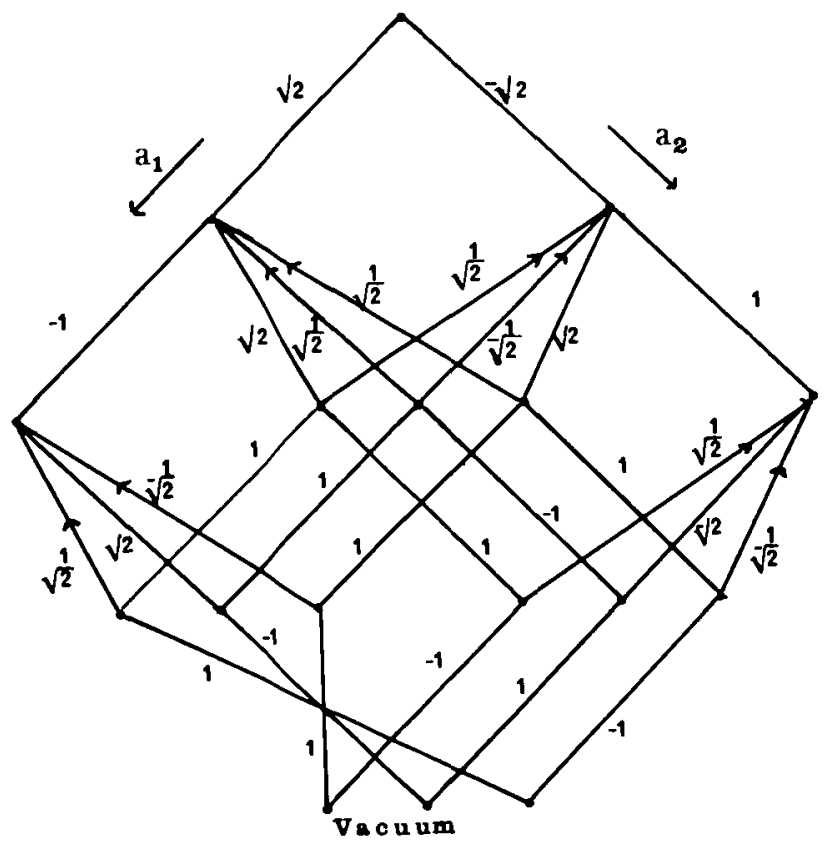

FiG. 1. An example of a degenerate vacuum representation. Each dot is an eigenstate of $N_{1}=a_{1}^{*} a_{1}$ and $N_{2}=a_{2}^{*} a_{2}$.

\section{Conclusions}

The most significant conclusions to be drawn from the above discussion concerns representations of (2.1) with a unique vacuum state. These have been seen to describe identical particles only. Furthermore, if we take the representation (2.6) as being the most natural of such representations, then we see that a 
peculiar new form of the exclusion principle emerges: namely that the number of particles in the totality of eigenstates is bounded. This contrasts with the usual form which says that the number of particles in each eigenstate is bounded whereas there still may be an infinite number in the totality of eigenstates.

\section{Acknowledgements}

The author would like to thank both Dr. M. Andrews and Dr. T. S. Santhanam for many illuminating discussions.

\section{Appendix}

Buchdahl [2] has considered a truncated oscillator in which the basic commutation relation between raising and lowering operators is given by

$$
\left[a, a^{*}\right]=1-M K \text {, }
$$

with $K^{2}=K \neq 0, K a=0$ and $M>1$ is an integer.

We shall show that upon reinterpretation of the raising and lowering operators as creation and annihilation operators, respectively, the irreducible representations of (A.1) are the same as those for the scheme

$$
\left[a, a^{*} a\right]=a, \quad a^{M}=0 \quad \text { and } \quad a^{l} \neq 0 \text { for } l<M .
$$

This is just the scheme (2.1) with $i=j=k$ and $M=p+1$.

We begin by proving the last two observations made at the beginning of Section 2.

First, equation (1.8) shows that $a_{l}\left|i_{1}, i_{2}, \ldots, i_{l}, \ldots\right\rangle$ is an eigenvector of $N_{1}, N_{2}, \ldots$ with eigenvalues $i_{1}, i_{2}, \ldots, i_{l}-1, \ldots$ and that $a_{l}^{*} \mid i_{1}$, $i_{2}, \ldots, i_{l}, \ldots>$ has the set $i_{1}, i_{2}, \ldots, i_{l}+1, \ldots ;$ the only exceptions to this evidently being when they vanish. Because of (2.2), this occurs for $a_{l} \mid i_{1}$, $i_{2}, \ldots, i_{l}, \ldots>$ only when $i_{l}=0$.

Secondly, let $\gamma$ be a normalized eigenvector of $N_{l}$ with eigenvalue $q$; then $0 \leqslant\left(a_{l} \gamma, a_{l} \gamma\right)=\left(\gamma, a_{l}^{*} a_{l} \gamma\right)=q$, showing that the eigenvalues of $N_{l}$ are nonnegative. Also, because of the action of $a_{l}, q, q-1, q-2, \ldots$ are eigenvalues of $N_{l}$. This sequence must terminate at zero, otherwise negative eigenvalues of $N_{l}$ will occur. Thus $q$ must be an integer.

We move on to our main result. Consider an irreducible representation $\Lambda$ of (A.2). We may write $\left[a, a^{*}\right]=1-M^{*}$, and need show only that $K$ is the required projection operator. First, we observe that $(1-M K) a=\left[a, a^{*}\right] a=$ $\left[a, a^{*} a\right]=a$ and so $K a=0$ as required. We now show that $K$ is projective. To 
begin with, we have

$$
\left[a a^{*}, a^{*} a\right]=a\left[a^{*}, a^{*} a\right]+\left[a, a^{*} a\right] a^{*}=-a a^{*}+a a^{*}=0 .
$$

This means that there is a basis for $\Lambda$ on which $a^{*} a$ and $a a^{*}$ are diagonal. Because of the second equation of (A.2), at least one of the basis vectors must have an eigenvalue of $M-1$ for $a^{*} a$. Let $\beta$ be a normalized basis vector with this property. Now define $\chi \subset \Lambda$ to be the subspace generated by the sequence of vectors

$$
\beta, a \beta, a^{2} \beta, \ldots, a^{M-1} \beta .
$$

The following lemma applies to $\chi$.

LEMMA 3. If $\eta$ belongs to the basis discussed above and

(i) if $\eta \in \chi$ then $a \eta \in \chi$ and $a^{*} \eta \in \chi$, and

(ii) if $\eta \notin \chi$ then a $\notin \chi$ or it is zero, and likewise for $a^{*} \eta$.

Proof. (i) The first part is obvious because $a^{M} \beta=0$. For the second part, $\eta$ may be written as $\eta=\sum_{i=0}^{M-1} c_{i}(a)^{\prime} \beta$, with $c_{i}$ being constants, and so

$$
a^{*} \eta=c_{0} a^{*} \beta+\sum_{i=1}^{M-1} c_{i}(M-1-i)(a)^{i-1} \beta .
$$

Now, if $a^{*} \beta \neq 0$, it is an eigenvector of $a^{*} a$ with value $M$, which means that $a^{M}\left(a^{*} \beta\right) \neq 0$, contradicting (A.2). Hence $a^{*} \eta \in \chi$.

(ii) Suppose $a \eta \neq 0 \in \chi$. Then $a^{*} a \eta=k^{\prime} \eta$ with $k^{\prime} \neq 0$. But $a^{*}(a \eta) \in \chi$ from (i), which is a contradiction since $\eta=\left(k^{\prime}\right)^{-1} a^{*} a \eta \notin \chi$. Similarly, suppose $a^{*} \eta \neq$ $0 \in \chi$. Then $a a^{*} \eta=k^{\prime \prime} \eta$ with $k^{\prime \prime} \neq 0$ because $\left\|a^{*} \eta\right\|=\left|\left(\eta, a a^{*} \eta\right)\right|^{1 / 2}$. But $a\left(a^{*} \eta\right) \in \chi$ from (i), which again is a contradiction because $\eta=\left(k^{\prime \prime}\right)^{-1} a a^{*} \eta \notin \chi$.

Define now the map $P$ as follows, with $\eta$ the same as in Lemma 3: $P \eta=\eta$ for $\eta \in \chi$ and $P \eta=0$ for $\eta \notin \chi$. It is clear that Lemma 3 implies that $[P, a]=$ $\left[P, a^{*}\right]=0$. Thus $P$ commutes with every element of the algebra for $a$ and $a^{*}$ and hence by Schur's Lemma must be a multiple of the identity. This is only possible if $\chi=\Lambda$.

Now, since the vectors of (A.3) are orthogonal, corresponding to different eigenvalues of $a^{*} a$, they may serve as a basis set for $\Lambda$. Thus, in order to show that $K$ is projective and non-zero, we need show only that $K \beta=\beta$, because $K(a)^{i} \beta=0$ for $i \geqslant 1$. From above we have $a^{*} \beta=0$ and so $(1-M K) \beta=$ $-a^{*} a \beta=(1-M) \beta$. Hence $K \beta=\beta$ as required.

Consider now an irreducible representation $\Lambda^{\prime}$ of (A.1), First, we have $\left[a, a^{*} a\right]=\left[a, a^{*}\right] a=(1-M K) a=a$. Thus it remains to be shown that the second equation of (A.2) holds on $\Lambda^{\prime}$. Now, as before, we have $\left[a a^{*}, a^{*} a\right]=0$, from which we deduce, with the help of (A.1), that $\left[K, a^{*} a\right]=\left[K, a a^{*}\right]=0$. This allows us to choose a basis for $\Lambda^{\prime}$ in which $a^{*} a, a a^{*}$ and $K$ are diagonal. 
Denote by $|n m k\rangle$ a normalized basis vector with $n, m$ and $k$ being the eigenvalues of $a^{*} a, a a^{*}$ and $K$, respectively. Using (A.1) we see that $K a^{*} a=M K^{2}-K$ $=(M-1) K$. Applying $|n m k\rangle$ to this equation we are led to

$$
k n=(M-1) k \text {. }
$$

Now $K$ is projective so $k=0,1$. Hence, for $k=1$, (A.4) shows that $n=M-1$. Applying $|n m k\rangle$ to (A.1) gives $m-n=1-M k$. This shows that, when $k=1$, $n=M-1$ and $m=0$. Since $K \neq 0$ there must therefore be a $|M-1,0,1\rangle$. Call one such vector $\beta^{\prime}$ and define the subspace $\chi^{\prime}$ of $\Lambda^{\prime}$ as that generated by the vectors

$$
\beta^{\prime}, a \beta^{\prime}, a^{2} \beta^{\prime}, \ldots, a^{M-1} \beta^{\prime} .
$$

Notice that $a^{M} \beta^{\prime}=0$ because of the fact that $\left[a, a^{*} a\right]=a$ holds on $\Lambda^{\prime}$.

Lemma 3, proved above for $\chi$, is easily seen to hold for $\chi^{\prime}$; the only non-trivial aspect is showing that $a^{*} \beta^{\prime}=0$. This can be seen to be true because

$$
\left\|a^{*} \beta\right\|=\left|\left\langle 1,0, M-1\left|a a^{*}\right| M-1,0,1\right\rangle\right|^{1 / 2}=0 .
$$

Defining a $P^{\prime}$ as $P$ was defined above, we are led as before to $\left[P^{\prime}, a\right]=\left[P^{\prime}, a^{*}\right]$ $=0$ and so, via Schur's Lemma, to the fact that $\chi^{\prime}=\Lambda^{\prime}$. From the remark made following (A.5), it is now trivial to see that the second of (A.2) holds on $\chi^{\prime}=\Lambda^{\prime}$.

\section{References}

[1] I. Bialynicki-Birula, "Elementary particles and generalized statistics", Nucl. Phys. 49 (1963), 605-608.

[2] H. A. Buchdahl, "Concerning a kind of truncated quantized linear harmonic oscillator", Amer. J. Phys. 35 (1967), 210-218.

[3] B. Geyer, "On generalized field quantization procedures", Nucl. Phys. B9 (1969), 67-74.

[4] A. B. Govorkov, in High-energy physics and elementary particle theony, Int. school for theoretical physics, Yalta, 1966 (Kiev, 1967), page 770 (in Russian).

[5] H. S. Green, "Generalized methods for field quantization", Phys. Rev. 90 (1953), 270-273.

[6] W. Pauli, "On the connection between spin and statistics", Prog. Theor. Phys. 5 (1950), 526-543.

[7] P. Roman and J. J. Aghassi, preprint BU-EP-5-66 (Boston University, 1966).

Department of Theoretical Physics

Faculty of Science

Australian National University

Canberra, A.C.T. 2600 